\title{
Increasing the Use of Animal-free Recombinant Antibodies
}

doi:10.14573/altex.2001071

\begin{abstract}
Antibodies are used in a range of research, diagnostic, and regulatory applications. Traditional methods for producing such reagents involve the immunization of animals, which introduces variability into the methods that use them and is not aligned with efforts to replace and reduce animal use. Experts from academia, biotechnology, government, and animal protection organizations met December 3, 2019, at the National Institutes of Health in Bethesda, MD, USA to discuss the status of development and use of animal-free recombinant antibodies and their potential to replace antibodies derived from animals. This paper summarizes the discussion and the actions that resulted to facilitate increased production and use of animal-free recombinant antibodies.
\end{abstract}

\section{Introduction}

Lack of reproducibility in biomedical research has reached crisis proportions, and antibodies from animal sources are one of the largest contributors to the problem (Baker, 2015; Bradbury and Plückthun, 2015; Berglund et al., 2008). As a result, scientists are calling for an international shift to using animal-free sequence-defined recombinant antibodies or other affinity reagents. Such reagents have numerous scientific benefits over animal-derived antibodies, including higher affinity and specificity, faster generation time, and reduced immunogenicity (Groff et al., 2015; Bradbury and Plückthun, 2015).

In December 2018, a working group of the Scientific Advisory Committee of the European Union Reference Laboratory for Alternatives to Animal Testing reviewed the scientific validity and benefits of animal-free technologies to produce affinity reagents. The working group concluded that use of animal-free recombinant antibodies would improve scientific reproducibility and that scientists should work toward the replacement of animal-derived antibodies (Barroso, 2019).

Building on the findings of the working group report, a December 3, 2019 expert meeting "Developing strategies to increase the use of recombinant antibodies" was convened by the National Toxicology Program Interagency Center for the Evaluation of Alternative Toxicological Methods (NICEATM) and the PETA International Science Consortium Ltd. at the National Institutes of Health in Bethesda, MD. The goal of the meeting was to outline a pathway to improve the quality and reproducibility of biomedical research by accelerating the production and use of animal-free recombinant antibodies in the United States. Discussions identified potential scientific and logistical hurdles and steps to overcome these barriers.

\section{Current needs for antibodies in research and testing}

Antibodies are one of the most widely used tools in laboratories and are crucial to a variety of applications across scientific disciplines. Recombinant antibodies are those with a defined sequence and include animal-free recombinant antibodies, antibodies derived from animal antibody libraries, and antibodies sequenced from hybridomas. Animal-free recombinant antibodies are defined here as those produced without the use of animals (e.g., derived from human antibody libraries).

Meeting participants identified the following characteristics that recombinant antibodies would need to have in order to achieve broader use throughout the scientific community.

\section{Interchangeable with currently used antibodies}

Animal-free recombinant antibodies would need to be incorporated effortlessly into currently used laboratory protocols and procedures. To be compatible with current uses, animal-free recombinant antibodies should be made available as purified proteins in the IgG format or with an Fc region instead of as DNA sequences or plasmids, and they should be amenable to post-translational modifications.

\section{Versatile}

Monoclonal antibodies, antibodies that recognize multiple epitopes, and functional and species cross-reactive antibodies are all needed for a variety of applications (e.g., western blot, immunoprecipitation, immunohistochemistry, immunofluorescence, flow cytometry). Production of animal-free recombinant antibodies would have to address these needs.

\section{Marketable}

Animal-free recombinant antibodies must be similarly priced to animal-derived antibodies and demonstrate equal or better analytical performance.

\section{Barriers to the use of animal-free recombinant antibodies}

Meeting participants identified the following challenges that have hindered adoption of animal-free recombinant antibodies, despite their scientific benefits. 


\section{Lack of awareness of the advantages of animal-free} recombinant antibodies

A growing number of publications have demonstrated both the wastefulness of animal-derived antibodies and the scientific capabilities of animal-free recombinant antibodies. However, there needs to be a concerted effort to show scientists how their research can benefit from making the switch.

\section{Lack of awareness of the current availability of animal-free recombinant antibodies}

While availability is still limited, some companies and institutions offer animal-free recombinant antibodies in formats ranging from DNA sequences to purified proteins that can be ordered from a catalog.

\section{Lack of availability of off-the-shelf animal-free recombinant antibodies for purchase}

The cost of developing a new animal-free recombinant antibody can be prohibitive for some users. Therefore, they need the option to purchase catalog animal-free recombinant antibodies at a similar price as animal-derived antibodies.

\section{Lack of quality antigens}

High quality antigens result in the production of higher performing antibodies (animal-derived or animal-free). Researchers should consider the type of epitope on the antigen (conformational or linear) and its location and the application in which the antibody will be used (denaturing or nondenaturing procedures). Antibody users also must be educated on the applicability domains of their antibodies.

\section{Next step actions to increase use of animal-free recombinant antibodies}

The objective of the meeting was to outline a pathway that will foster a large-scale transition to animal-free recombinant antibodies. With this in mind, meeting participants considered tangible activities and approaches that would have the greatest impact, concentrating on antibodies produced for basic research, which represent the largest antibody market.

\section{Phase out approach}

With the goal of eliminating the use of animals in the production of all types of antibodies, initial steps would address the most problematic practices and include: 1) a phased timeline to immediately eliminate the most inhumane and unnecessary production method of animal-derived antibodies - the ascites method - and 2) an agreement to end the continued production and use of scientifically flawed animal-derived polyclonal antibodies and replace these with validated animal-free recombinant counterparts in the near term.

\section{Prioritizing}

There are two possible approaches to begin to replace animal-derived antibodies: 1) developing new animal-free recombinant antibodies for targets that do not have already-developed ani- mal-derived antibodies, and 2) converting the most commonly used animal-derived antibodies (e.g., antibodies for housekeeping proteins, epitope tags, cell-specific markers, disease-specific markers, and basic cell signaling pathways) to animal-free recombinant antibodies, focusing first on those that do not meet validation standards. To facilitate the conversion of animal-derived antibodies that do not meet validation standards, a list of commonly ordered antibodies should be created and prioritized for recombinant development.

\section{Validation}

Thousands of animal-free recombinant antibodies have been developed, but many have not been validated for use in specific applications. Further, validation standards have not been adopted. There is a need to review suggested minimum standards for validation (e.g., Uhlen et al., 2016; Bourbeillon et al., 2010) and come to a consensus on what criteria researchers should use and journals should require - when conducting and publishing research using antibodies.

\section{Education}

A central resource with information on animal-free recombinant antibodies, the ability to directly purchase from corporate and academic suppliers, and educational materials such as webinars, classes, and hands-on workshops would facilitate the uptake of this technology. This resource would also connect to existing repositories of sequenced antibodies, such as the ABCD (AntiBodies for Chemically Defined) database and the Arizona State University's DNASU plasmid repository, which stores and distributes plasmids to researchers worldwide, including clones used for recombinant antibody production. Such repositories save sequences from being lost and, when the sequences are publicly available, give researchers the opportunity to engineer the antibodies, modify them, and test them in applications. A central resource for information on animal-free recombinant antibodies would need a network of experts to maintain.

\section{Partnerships}

Public-private partnerships among companies, academics, non-governmental organizations (NGOs), and federal and state government agencies would expedite the adoption of animal-free recombinant antibodies. In partnerships, different sectors can leverage their unique capabilities. For example, university researchers and small companies may specialize in the discovery of new antibodies, while large companies may excel in validation, scaling-up production, and distributing antibodies. This is exemplified in the Recombinant Antibody Network (RAN), composed of centers at the University of Chicago, University of Toronto, and University of California San Francisco, and Absolute Antibody Ltd.'s recent partnership to make more than 200 RAN recombinant antibodies more widely accessible (Absolute Antibody, 2019).

\section{Central facility}

Due to the expertise needed to successfully operate a recombinant antibody laboratory, a centralized facility would be more effective than individual universities or federal laboratories op- 
erating separate facilities. A central facility would require a large network of researchers to utilize its services and a long-term commitment to its funding.

\section{Funding}

Government agencies, NGOs, and universities could provide funding for various purposes, such as an informational resource on animal-free recombinant antibodies, partnerships, a central facility, and validation. Another approach would be to provide supplementary funding for awarded research projects to develop or purchase animal-free recombinant antibodies, or funding the development of animal-free recombinant antibodies for common targets. U.S. government agencies could also provide funding for animal-free recombinant antibody development and production to small companies through the Small Business Innovation Research program.

\section{Other incentives}

Outside forces or incentives will be required to overcome inertia and stimulate a sea change in antibody use. The following incentives were discussed at the expert meeting:

Regulatory agencies could fast-track the acceptance of diagnostic or other tests or therapies using animal-free recombinant antibodies.

A market branding strategy could be developed to identify and promote animal-free recombinant antibodies.

Journals could require that antibodies used in published research be sequenced, at a minimum, to promote more reproducible research.

\section{Conclusions}

No longer must the scientific community accept poorly characterized antibodies and the inherent risk that they bring to research. Meeting participants identified promising activities to overcome hurdles that have slowed the adoption of a tool that will improve scientific rigor and eliminate waste. The identified activities will increase awareness about the capabilities of animal-free recombinant antibodies and currently available resources and, with multi-sector commitments, will increase funding and infrastructure for their development and validation. The meeting conveners have committed to facilitating agreed upon next steps, and plans are underway to develop a strategy to accomplish the above tasks in the coming year.

\section{References}

Absolute Antibody (2019). Absolute Antibody partners with the Recombinant Antibody Network to facilitate access to engineered recombinant antibodies. 3 December 2019 press release. https://absoluteantibody.com/wp-content/uploads/2019/ 11/RAN-partnership-PR-FINAL.pdf
Baker, M. (2015). Reproducibility crisis: blame it on the antibodies. Nature 521, 274-276. doi:10.1038/521274a

Barroso, J. (2019). Scientific validity of replacements for animal-derived antibodies (presentation). Arlington, VA, USA: Scientific Advisory Committee on Alternative Toxicological Methods Meeting. https://ntp.niehs.nih.gov/ntp/about_ntp/ sacatm/2019/september/presentations/1-4-barroso-508.pdf

Berglund, L., Bjorling, E., Oksvold, P. et al. (2008). A genecentric human protein atlas for expression profiles based on antibodies. Mol Cell Proteomics 7, 2019-2027. doi:10.1074/mcp. R800013-MCP200

Bourbeillon, J., Orchard, S., Benhar, I. et al. (2010). Minimum information about a protein affinity reagent (MIAPAR). Nat Biotechnol 7, 650-653. doi:10.1038/nbt0710-650

Bradbury, A. and Plückthun, A. (2015). Reproducibility: standardize antibodies used in research. Nature 518, 27-29. doi:10. $1038 / 518027 \mathrm{a}$

Groff, K., Brown, J. and Clippinger, A. J. (2015). Modern affinity reagents: recombinant antibodies and aptamers. Biotechnol Adv 33, 1787-1798. doi:10.1016/j.biotechadv.2015.10.004

Uhlen, M., Bandrowski, A., Carr, S.et al. (2016). A proposal for validation of antibodies. Nat Methods 13, 823-827. doi: 10.1038/nmeth.3995

\section{Conflict of interest}

The authors declare they have no conflicts of interest.

\section{Acknowledgements}

The meeting organizers thank the experts who attended the December 3, 2019 workshop for their discussions: A. Bradbury (Specifica), C. Chandrasekera (Canadian Centre for Alternatives to Animal Methods), P. Cosson (University of Geneva), C. DeWit (MilliporeSigma), S. Dübel (Technische Universität Braunschweig, YUMAB), J. Elliot (National Institute of Standards and Technology), A. Gray (Afability), H. Hamadeh (Genmab), B. Kay (University of Illinois at Chicago), A. Kossiakoff (University of Chicago), A. Solache (Abcam), K. Sullivan (Physicians Committee for Responsible Medicine), S. Sidhu (Toronto Recombinant Antibody Centre), and J. Wells (University of California San Francisco). Thanks also to Catherine Sprankle and Amber Daniel (ILS, contractors supporting NICEATM) for their editorial support.

Katherine Groff ${ }^{1}$, David Allen ${ }^{2}$, Warren Casey $^{3}$ and Amy J. Clippinger ${ }^{1}$

\footnotetext{
1PETA International Science Consortium Ltd., London, United Kingdom; 2Integrated Laboratory Systems, Research Triangle Park, NC, USA; ${ }^{3}$ National Toxicology Program Interagency Center for the Evaluation of Alternative Toxicological Methods, National Institute of Environmental Health Sciences, Research Triangle Park, NC, USA
} 